\title{
ПЕРВЫЙ ОПЫТ ИСПОЛЬЗОВАНИЯ ТРЕХМЕРНОЙ СИСТЕМЫ ВИЗУАЛИЗАЦИИ NGENUITY® 3D VISUALIZATION SYSTEM В ХИРУРГИИ КАТАРAKT С ИМПЛАНТАЦИЕЙ PRE-LOADED ИНТРАОКУЛЯРНЫХ ЛИНЗ ACRYSOF IQ ULTRASERT
}

\author{
FIRST EXPERIENCE OF USING THREE-DIMENSIONAL VISUALIZATION SYSTEM \\ NGENUITY 3D VISUALIZATION SYSTEM IN SURGERY OF CATARACT WITH \\ IMPLANTATION OF PRE-LOADED INTRAOCULAR LENS ACRYSOF IQ ULTRASERT
}

\section{Стебнев В.С. 1 Стебнев С.Д. ${ }^{2}$ Малов И.В.1}

\author{
1ФГБОУ ВО «Самарский государственный \\ медицинский университет» Минздрава России \\ 2000 «Хирургия глаза» офтальмологическая \\ клиника, Самара
}

\begin{abstract}
Цель - оценить клиническую эффективность и особенности использования новой трехмерной системы визуализации NGENUITY в хирургии катаракт с имплантацией pre-loaded интраокулярных линз AcrySof IQ Ultrasert (ALCON).
\end{abstract}

Материалы и методы. Изучены клинические результаты использования 3D-системы визуализации NGENUITY в хирургии катаракт у 100 пациентов (100 глаз) в возрасте $64,3 \pm 6,1$ года; женщин - 83, мужчин - 17. Имплантированы монофокальные ИОЛ AcrySof IQ Ultrasert компании Alcon с расчетом на рефракцию цели $\mathrm{Em} \pm 0,5$ дптр с использованием навигационной системы VERION (Alcon).

Результаты. Интраоперационных и послеоперационных осложнений не было. Осмотр пациентов проводился на 1 и 7 сутки и через 1 и 3 месяца после операции. Послеоперационный период протекал гладко, без инъекций. В результате лечения некорригированная острота зрения вдаль составила $0,74 \pm 0,02$ (от 0,2 до 1,0$)$ и зависела от состояния глазного дна. Средний сфероэквивалент клинической рефракции $0,38 \pm 0,47$ дптр. Рефракция цели достигнута у $91 \%$ больных. Потеря клеток заднего эпителия роговицы составила $3,8 \pm 1,5 \%$.

Результаты использования 3D-системы визуализации NGENUITY в хирургии катаракт позволяют считать, что она имеет ряд существенных преимуществ по сравнению с применением традиционных методов современной микрохирургии.

Выводы. 1. Новая трехмерная система визуализации NGENUITY ${ }^{\circledR}$ 3D Visualization System обеспечивает хирургу значительно больше возможностей, чем просто улучшенная визуализация.

\section{Stebnev VS1 \\ Stebnev SD ${ }^{2}$ \\ Malov IV 1}

'Samara State

Medical University

2 "Eye Surgery" ophthalmologic

clinic, Samara

Aim - to evaluate the clinical efficacy and features of use new three-dimensional visualization system "NGENUITY" in surgery of cataract with implantation of "pre-loaded" intraocular lens "AcrySof IQ Ultrasert" (ALCON).

Materials and methods. We investigated the clinical results of using a $3 \mathrm{D}$ visualization system "NGENUITY" cataract surgery in 100 patients (100 eyes) age of $64.3 \pm 6.1$ years; 83 women and 17 men. Implanted monofocal IOLs "AcrySof IQ Ultrasert" company "Alcon" with a goal refraction Em $\pm 0,5$ diopters with the use of navigation systems "VERION" (Alcon).

Results. There were no intraoperative or postoperative complications. Examination of patients was carried out on 1 and 7 days and 1 and 3 months after surgery. The postoperative period was smooth, without injections. As a result of treatment, uncorrected visual acuity in the distance was $0.74 \pm 0.02$ ( 0.2 to 1.0$)$ and depended on the fundus condition. The average sphericules clinical refraction $0,38 \pm 0,47$ dioptres. Refraction of the target was achieved in $91 \%$ of patients. Loss of corneal posterior epithelial cells was $3.8 \pm 1.5 \%$.

The results of the use of $3 \mathrm{D}$ imaging system "NGENUITY" in cataract surgery suggest that it has a number of significant advantages over the use of traditional methods of modern microsurgery.

Summary. 1. A new three-dimensional visualization system "NGENUITY 3D Visualization System" provides the surgeon much more than just improved visuals. 
2. Практическое освоение и дальнейшее технологическое развитие системы NGENUITY ${ }^{\circledR}$ 3D Visualization System открывает новые научные и практические перспективы в хирургии катаракт.

3. Использование новой системы ULTRASERT Pre-loaded IOL Delivery System при имплантации монофокальных ИОЛ существенно оптимизирует хирургию катаракт.

4. Новые технологические особенности конструкции системы ULTRASERT Pre-loaded IOL Delivery System позволяют исключить риск инфицирования и повреждения линз, обеспечивают быстрое и стабильное их размещение в капсульном мешке, гладкое течение послеоперационного периода.

Ключевые слова: 3D-хирургия катаракт, NGENUITY, катаракта, PRE-LOADED, интраокулярные линзы, ACRYSOF IQ, ULTRASERT.

Конфликт интересов: не заявлен.
2. Practical development and further technological development of the system "NGENUITY 3D Visualization System" opens new scientific and practical prospects in cataract surgery.

3. The use of the new system "ULTRASERT Preloaded IOL Delivery System" the implantation of a monofocal IOL significantly optimizes the surgery of cataracts.

4. New technological features of the system design "ULTRASERT Pre-loaded IOL Delivery System" enable to eliminate the risk of infection and damage to the lens, allow quick and stable placement in the capsular bag, a smooth postoperative period.

Keywords: 3D cataract surgery, NGENUITY, cataract, "PRE-LOADED" INTRAOCULAR LENS "ACRYSOF IQ", "ULTRASERT»

Conflicts of Interest: nothing to declare.

\section{ВВЕДЕНИЕ}

Гехнологический прогресс в офтальмохирургии затронул ее важнейший аспект - систему визуализации. Появившаяся возможность преобразовывать оптическое изображение от операционного микроскопа в цифровую систему визуализации обеспечивает беспрецедентное повышение качества трехмерного изображения операционного поля, проецируемого на экран [1, 2]. Первоначально в хирургии катаракты использовалась цифровая система TrueVision Systems [3]. Улучшение с годами характеристик используемых камер, применение более современного процессора с мощными видеокартами и дисплея высокого разрешения привело к созданию 3D-системы NGENUITY. Эта система явилась продуктом совместной работы двух компаний - Alcon и Truevision 3D Surgical. Eе составляют: камера высокого динамического разрешения (High Dynamic Range), совмещенная с оптикой операционного микроскопа, 55-дюймовый 3D-дисплей высокого разрешения (Ultra-HD 4K OLED), высокоскоростной графический процессор и пассивные поляризованные очки для трехмерного восприятия изображения. Все это позволило значительно оптимизировать хирургию катаракты [4] и использовать цифровую систему визуализации в хирургии сетчатки [5].

Продолжающееся совершенствование хирургии катаракты затронуло и такой немаловажный аспект, как оптимизация средств доставки интраокулярных линз (ИОЛ) в глаз. Инжекторный метод, быстро доказав свои преимущества, окончательно вытеснил использование пинцетов для введения ИОЛ в капсульный мешок. Сейчас передовыми производителями сделан новый оригинальный технологический шаг в разработке средств доставки линз в глаз - создание одноразовой моноблочной системы pre-loaded, включающей в себя инжектор, картридж и уже «загруженную» ИОЛ [6]. Клинический опыт использования этих систем показал их неоспоримые достоинства при имплантации ИОЛ: сокращение сроков операции, одноразовость использования, бесконтактная техника имплантации, минимальные сроки для подготовки линзы к имплантации, высокий уровень стерильности линз, плавное их продвижение и сворачивание с последующим предсказуемым расправлением в капсульном мешке, отсутствие риска повреждения оптической и гаптической части ИОЛ и отсутствие потенциальных ошибок при их загрузке [7].

\section{ЦЕЛЬ}

Оценить клиническую эффективность и особенности использования новой трехмерной системы визуализации NGENUITY в хирургии катаракт с имплантацией pre-loaded интраокулярных линз AcrySof IQ Ultrasert (ALCON).

\section{МАТЕРИАЛЫ И МЕТОДЫ}

С февраля 2018 года мы начали использовать новую систему - ULTRASERT Pre-loaded IOL Delivery System (Alcon) для имплантации монофокальных ИОЛ AcrySof IQ, появившуюся в Российской Федерации. Ранее система ULTRASERT была одобрена к клиническому применению в США, а с 2015 года - в Европе.

С июня 2018 года нами используется новая 3Dсистема визуализации NGENUITY® ${ }^{\circledR}$ D Visualization System компании Alcon при выполнении факоэмульсификации катаракт (ФЭК) с имплантацией интраокулярных линз (ИОЛ).

В исследование вошли 100 пациентов (100 глаз) в

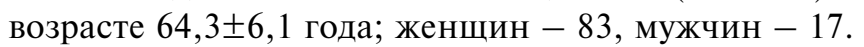
Все пациенты имели осложненные и возрастные катаракты различной степени зрелости и плотности ядер. До- и послеоперационное обследование включало визометрию, биомикроскопию переднего и заднего отрезков глаза, оптическую биометрию, пахиметрию, пневмотонометрию, кераторефрактометрию, эндотелиальную микроскопию, топографию роговицы, оптическую когерентную томографию макулярной зоны, исследование на диагностической системе VERION Image Guided System.

В процессе хирургии пациентам были имплантированы ИОЛ компании Alcon: AcrySof IQ Ultrasert(100). При поступлении: острота зрения от светоощущения до 0,7 , средняя максимально корригированная острота

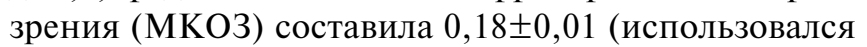




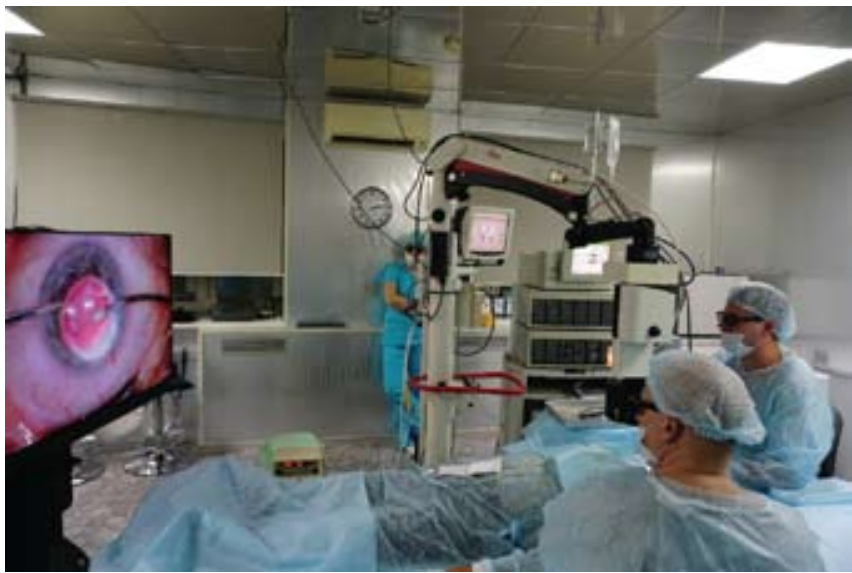

Рисунок 1. Профрессор кафедры глазных болезней ИПО СамГМУ д.м.н. В.С. Стебнев выполняет 3D-хирургию катаракты.

электронный фороптер HUVITZ HDR-7000, Южная Корея); ВГД 10,7 $\pm 3,8$ мм рт. ст. (бесконтактный тонометр REICHERT, CША); передне-задняя ось глаз по данным оптической и ультразвуковой биометрии была от 21,32 до 24,41 мм. Рефракция роговицы -

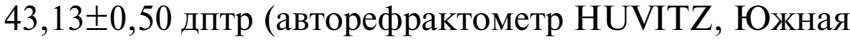
Корея). Состояние эндотелия роговицы оценивалось бесконтактным эндотелиальным микроскопом ТОМЕY EM-3000 (Япония) в центральной зоне и в 6 точках средней периферии: средняя плотность эндотелиальных клеток (ПЭК) составила $2445 \pm 244,7$ на мм².

Расчеты ИОЛ проводились на оптическом LENSTAR LS 900 (Швейцария) или ультразвуковом TOMEY (Япония) биометрах с использованием навигационной системы VERION Image Guided System на рефракцию цели $\mathrm{Em} \pm 0,5$ дптр или миопию слабой степени с учетом профессии пациента и его предпочтений.

В работе использованы микроскоп LEICA M844 (Germany) с камерой NGENUITY вместо оптических окуляров и хирургические системы CONSTELLATION (Alcon) и MILLENNIUM (Bausch\&Lomb).

Всем пациентам амбулаторно выполнена факоэмульсификация (ФЭК) под эпибульбарной анестезией по традиционным микрокоаксиальным технологиям Phakochope и Quickchope. Во всех случаях использовались вискоэластики Viscoat и ProVisc (ALCON), сбалансированный физиологический раствор BSS+.

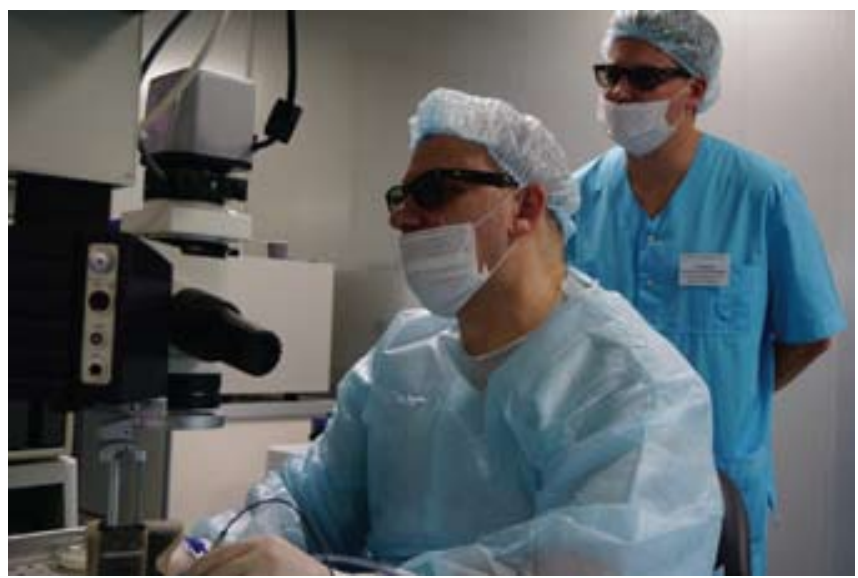

Рисунок 2. Д.м.н. С.Д. Стебнев для хирургии катаракт использует камеру NGENUITY вместо оптических окуляров микроскопа.

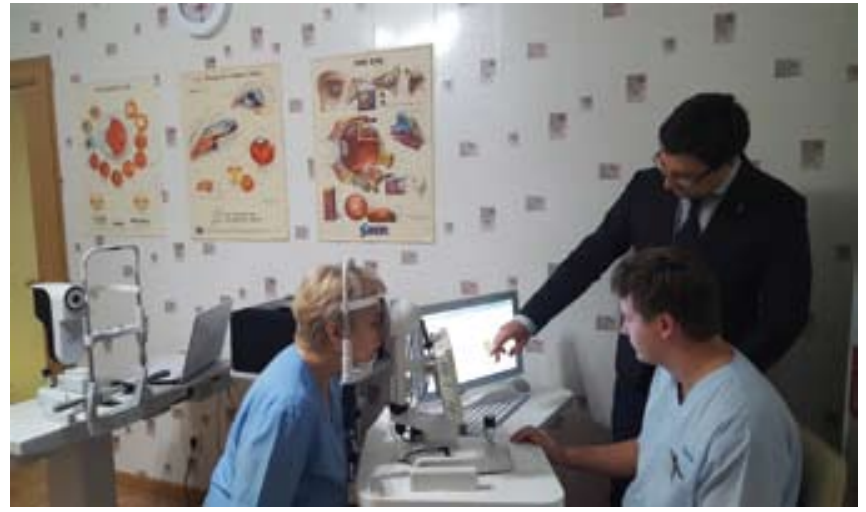

Рисунок 3. Заведующий кафедрой глазных болезней ИПО СамГМУ д.м.н. И.В. Малов и профрессор кафедры глазных болезней ИПО СамГМУ д.м.н. В.С. Стебнев проводят расчет интраокулярной линзы pre-loaded AcrySof IQ Ultrasert (ALCON) на системе VERION Image Guided System.

Для использования системы Ultrasert проводился рекомендуемый компанией Alcon чисто роговичный самогерметизирующийся разрез 2,2 мм. Использование системы Ultrasert начиналось с заполнения «Вискотом» ее контейнера, в котором располагалась ИОЛ, и удаления фиксирующих элементов. Затем под давлением плунжера линза продвигалась по картриджу и сворачивалась, после чего проводилась ее имплантация в капсульный мешок.

\section{— РЕЗУЛЬТАТЫ И ОБСУЖДЕНИЕ}

Интраоперационных и послеоперационных осложнений не было.

Осмотр пациентов проводился на 1 и 7 сутки и через 1 и 3 месяца после операции. Послеоперационный период протекал гладко, без применения инъекций. Использовались инстилляции стероидных и нестероидных препаратов, антисептиков. Через 3 месяца после операции некорригированная острота зрения вдаль в среднем составила $0,74 \pm 0,02$ (от 0,2 до 1,0 ) и зависела от состояния глазного дна. Средний сфероэквивалент клинической рефракции $0,38 \pm 0,47$ дптр. Рефракция цели достигнута у $91 \%$ больных. Потеря клеток заднего эпителия роговицы составила $3,8 \pm 1,5 \%$.

Первые результаты использования новой 3Dсистемы визуализации NGENUITY в хирургии катаракт позволили нам отметить ряд особенностей:

- мобильная конструкция новой системы позволяет правильно и комфортно для хирурга разместить дисплей в операционной [8];

- камера монтируется быстро в стандартное посадочное место после удаления окуляров микроскопа; дополнительные опции (лазерный фильтр и операционный модуль VERION) сохранены и могут быть использованы;

- адаптация хирурга к новой системе визуализации прошла быстро и без особых затруднений;

- существенно улучшилась эргономика рабочего места хирурга. Отсутствие вынужденной привязанности к окулярам микроскопа позволяет хирургу принимать анатомически правильное и удобное положение тела, что снижает риск развития у него нарушений опорнодвигательного аппарата [9]; 


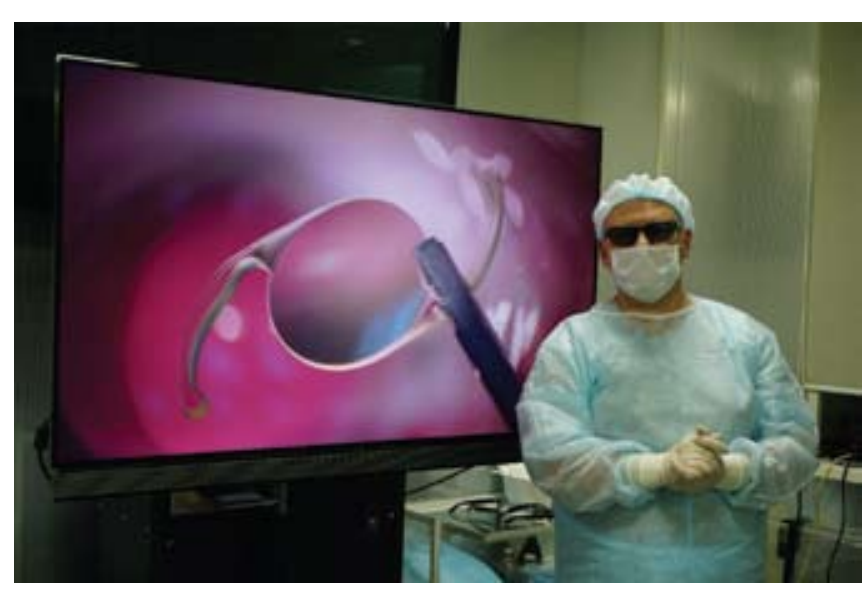

Рисунок 4. В операционной - д.м.н. С.Д. Стебнев За его спиной на экране широкопольное (55 дюймов) трехмерное отображение интраокулярной линзы AcrySof IQ Ultrasert.

- 3D-хирургия, по нашим субъективным ощущениям, более комфортна, ведет к меньшей общефизической усталости и снижению зрительной нагрузки хирурга; нами не отмечены симптомы 3D-астенопии [10];

- время выполнения первых 10 операций увеличилось на 3-4 минуты, последующие же операции по продолжительности не превышали их исполнения с окулярами микроскопа;

- присутствующие в операционной персонал и врачи кафедры глазных болезней ИПО СамГМУ, используя поляризованные очки, имели прекрасную возможность трехмерного восприятия всего хода операции;

- цифровые технологии новой системы визуализации позволяют достигать высокого уровня увеличения операционного поля без потери его четкости и контрастности; существенно увеличивается глубина резкости изображения (стереопсис);

- широкопольное (55 дюймов) трехмерное отображение операционного поля позволяет с высокой точностью выполнять все этапы операции (разрезы, капсулорексис, полировка задней капсулы, центрация ИОЛ и т.д.);

- использование различных цветных фильтров новой системы позволяет существенно расширить практические и научные аспекты катарактальной хирургии [11];

- высокая чувствительность камеры позволила нам использовать невысокий уровень освещенности операционного поля, что более комфортно для пациента и снижает риск ятрогенной ретинальной фототоксичности [12];

- в своей практике мы не испытывали затруднений с задержкой между манипуляциями рук хирурга и движениями изображения на мониторе;

- новая система позволяет выполнять 3D/2Dвидеозаписи и фоторегистрацию высочайшего качества.

Первые результаты использования ULTRASERT Pre-loaded IOL Delivery System позволили нам отметить высокий уровень комфорта, который испытывал хирург при имплантации ИОЛ, и выделить следующие инновационные особенности новой системы:

- удобный шприцеподобный дизайн новой системы, позволяющий хирургу имплантировать ИОЛ одной

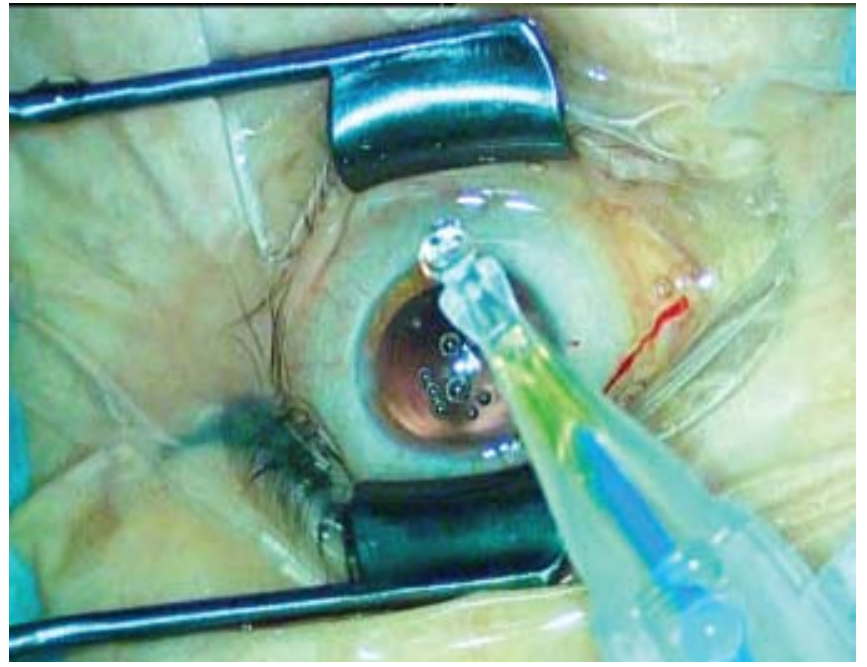

Рисунок 5. Момент имплантации монофокальной ИОЛ с использованием системы ULTRASERT Pre-loaded IOL Delivery System.

рукой и высвободить вторую руку для фиксации глазного яблока, а затем и для контроля расправления ИОЛ;

- герметичная конструкция контейнера, в котором находится ИОЛ до самого последнего момента ее имплантации, исключает возможный контакт ее с инструментами, воздухом и поверхностью глаза;

- возможность использовать широко известную для расчетов и манипуляций модель ИОЛ - AcrySof IQ;

- быстрые и простые манипуляции для подготовки системы к использованию;

- удобство нового запатентованного пружинного механизма поршня (TensionGlide), обеспечивающего его легкое скольжение и плавное продвижение ИОЛ;

- два хорошо заметных цветных стопора движения линзы и поршня, позволяющие обезопасить хирурга от случайных манипуляций;

- овальная форма наконечника насадки (Depth Guard) и оригинальный ограничитель глубины его введения в переднюю камеру, снижающие травмирование роговичного тоннеля размером 2,2 мм и сохраняющие его профиль в момент продвижения наконечника;

- выход поршня до 6,5 мм обеспечивает полное перемещение ИОЛ в переднюю камеру, а затем и в капсульный мешок;

- новый дизайн наконечника поршня, обеспечивающий высокий уровень предсказуемости сворачивания и расправления оптической и гаптической частей ИОЛ.

Таким образом, первый опыт использования новой 3D-системы визуализации NGENUITY в хирургии катаракт позволяет нам считать, что она имеет ряд существенных преимуществ по сравнению с традиционной современной микрохирургией. Дальнейшее развитие компьютерных технологий, возможно, будет способно обеспечить создание новых перспективных программ для системы NGENUITY, интегрировать в нее до- и интраоперационные цифровые изображения диагностических обследований, что обеспечит еще более высокие результаты лечения пациентов с катарактой. Первые имплантации монофокальных ИОЛ с использованием новой системы ULTRASERT Pre-loaded IOL Delivery System показали высокий технологический 
уровень и перспективность ее использования в клинике. Эту систему можно рассматривать как следующий шаг технологического совершенствования доставки и размещения ИОЛ в капсульном мешке. Система ULTRASERT Pre-loaded IOL Delivery System, вероятно, должна полностью вытеснить применение инжекторов и картриджей и стать таким образом новым стандартом в имплантации ИОЛ.

\section{ВЫВОДЫ}

1. Новая трехмерная система визуализации NGENUITY ${ }^{\circledR}$ 3D Visualization System обеспечивает хирургу значительно больше возможностей, чем просто улучшенная визуализация.

\section{ЛИТЕРАТУРА / REFERENCES}

1. Chang D. An embarrassment of riches. Cataract \& Refractive Surgery Today. 2008;8:7-10.

2. Dutra-Medeiros M, Nascimento J, Henriques J, Barrao S. Three-dimensional head-mounted display system for ophthalmic surgical procedures. Retina. 2017;37:1411-1414.

3. Weinstock R. Heads up cataract surgery using high-definition 3-D microscopic viewing system. Paper presented at: American Society of Cataract and Refractive Surgery Annual Meeting; April 3-8, 2009; San Francisco, CA.

4. Weinstock R. Prospective study of 3D image guidance templates during small-incision cataract surgery. Paper presented at: American Society of Cataract and Refractive Surgery Annual Meeting; March 25-29, 2011; San Diego, CA.

5. Eckardt C. Live surgery presentation in 3-D. Frankfurt Retina Meeting; March 15; Mainz, Germany, 2014.

6. Wang L, Wolfe P, Chernosky A, Paliwal S. In vitro delivery performance assessment of a new preloaded intraocular lens delivery system. J Cataract Refract Surg. 2016;42(12):18141820 .
2. Практическое освоение и дальнейшее технологическое развитие системы NGENUITY ${ }^{\circledR} 3 \mathrm{D}$ Visualization System открывает новые научные и практические перспективы в хирургии катаракт.

3. Использование новой системы ULTRASERT Preloaded IOL Delivery System при имплантации монофокальных ИОЛ существенно оптимизирует хирургию катаракт.

4. Новые технологические особенности конструкции системы ULTRASERT Pre-loaded IOL Delivery System позволяют исключить риск инфицирования и повреждения линз, обеспечивают быстрое и стабильное их размещение в капсульном мешке, гладкое течение послеоперационного периода.

7. Chung B, Lee H, Choi M, Seo K. Preloaded and non-preloaded intraocular lens delivery system and characteristics: human and porcine eyes trial. Int J Ophthalmol. 2018;11(1):6-11.

8. Romano M, Cennamo G, Comune C, Cennamo M. Evaluation of 3D heads-up vitrectomy: outcomes of psychometric skills testing and surgeon satisfaction. Eye (Lond). 2018;32:10931098 .

9. Marx J, Wertz F, Dhimitri K. Work-related musculoskeletal disorders in ophthalmologists. Techniques in Ophthalmology. 2005;3:54-61.

10. Chow D. The effect of light source filters on tissue visualization: a multicenter trial. Euretina 13th Congress; September 2013; Hamburg.

11. Kim S, Suh Y, Song J, Park J. Clinical research on the ophthalmic factors affecting 3D asthenopia. Ophthalmol Strabismus. 2012;49:248-253.

12. Youssef $\mathrm{P}$, Sheibani N, Albert D. Retinal light toxicity. Eye (Lond). 2011;25:1-14.

Конфликт интересов: все авторы заявляют

об отсутствии потенциального конфликта интересов,

требующего раскрытия в данной статье.

\section{Автор для переписки}

Стебнев Вадим Сергеевич

Адрес: кафедра глазных болезней ИПО СамГМУ,

ул. Ташкентская, 159, г. Самара, Россия, 443095.

E-mail: vision63@yandex.ru

Тел.: +7 (919) 8099647 .

\section{Corresponding Author}

Stebnev Vadim Sergeevich

Address: Department of ophthalmology of

Institute of Samara State Medical University,

195 Tashkentskaya st., Samara, Russia, 443095.

E-mail: vision63@yandex.ru

Tel: + 7 (919) 8099647.

\section{СВЕДЕНИЯ ОБ АВТОРАХ}

Стебнев В.С. - Д.М.Н., доцент, профессор кафедры глазных болезней ИПО СамГМУ. E-mail: vision63@yandex.ru

Стебнев С.Д. - д.м.н., директор офтальмологической клиники ООО «Хирургия глаза» (г. Самара). E-mail: stebnev2011@yandex.ru

Малов И.В. - д.м.н., професссор, заведующий кафедрой глазных болезней ИПО СамГМУ.

E-mail: ivmsamara@gmail.com

\section{INFORMATION ABOUT AUTHORS}

Stebnev VS - PhD, associate Professor, professor in the Department of eye diseases, Institute of Samara State Medical University. E-mail: vision63@yandex.ru

Stebnev SD - PhD Director

of the eye clinic, "Eye Surgery" (Samara) E-mail: stebnev2011@yandex.ru

Malov IV - PhD, Professor, head of Department of eye diseases, Institute of Samara State Medical University. E-mail: ivmsamara@gmail.com 